\title{
A theoretical study of an electrostatic multipole to assess the horizontal and vertical distribution of the in situ water content of vegetation canopies
}

\author{
Jérôme HELBERT ${ }^{\mathrm{a} *}$, Paul BoISSARD ${ }^{\mathrm{b}}$, Jean-Pierre BuIS ${ }^{\mathrm{c}}$ \\ ${ }^{a}$ Institut National de la Recherche Agronomique, Unité Environnement et Grandes Cultures, 78850 Thiverval Grignon, France \\ ${ }^{\mathrm{b}}$ Institut National de la Recherche Agronomique, Unité de Recherches Intégrées en Horticulture, Route des Colles, \\ Sophia-Antipolis, 06410 Biot, France \\ ${ }^{\mathrm{c}}$ CIMEL Électronique, 172 rue de Charonne, 75011 Paris, France
}

(Received 21 March 2000; revised 9 October 2000; accepted 26 October 2000)

\begin{abstract}
A theoretical study of an electrostatic multipole is presented. This instrument allows the non-destructive and in situ estimation of the water content of vegetation canopy horizontal layers from a measurement of their dielectric permittivities. The multipole is composed of four electrodes, two electrodes injecting an alternating current, while the two others measure a potential difference. A prototype instrument showed that good estimations of the wheat spike water content could be achieved. A simulation based on the finite element method is performed to study the response of the instrument for various wheat crop dielectric properties. Results show that the potential difference depends on the dielectric properties of the spikes and the stems, and that the soil influence is not significant. An improved configuration of the instrument, using two pairs of reception electrodes, is also simulated in order to be able to retrieve the spike and the stem permittivities. The inversion of the potential differences is realized by using an artificial neural network. The spikes' and the stems' permittivities can then be retrieved with a good accuracy (variances on estimated permittivities less than 0.01).
\end{abstract}

water content / dielectric permittivity / non-destructive method / vegetation canopy / biomass

Résumé - Étude théorique d'un multipôle électrostatique permettant d'accéder in situ à la distribution horizontale et verticale du contenu en eau des couverts végétaux. Cet article présente une étude théorique d'un multipôle électrostatique. Cet instrument permet une estimation in situ et non destructive du contenu en eau des strates horizontales d'un couvert végétal grâce à la mesure de leur permittivité diélectrique. Le multipôle est composé de quatre électrodes, deux électrodes injectant un courant alternatif tandis que les deux autres mesurent une différence de potentiel. Un premier prototype a montré la validité de la méthode pour estimer le contenu en eau des épis de blé. Un travail de simulation basé sur la méthode des éléments finis a permis d'étudier précisément la réponse de l'instrument en fonction des propriétés diélectriques d'une culture de blé. Les résultats montrent que la différence de potentiel dépend à la fois des propriétés des épis et des tiges, et que l'influence du sol n'est pas significative. Une version améliorée du quadripôle, comportant deux paires d'électrodes de réception, est alors simulée afin de pouvoir retrouver la permittivité des épis et celle des tiges. L'inversion des différences de potentiel est réalisée à l'aide d'un réseau de neurones artificiels. La précision obtenue est bonne, la variance des permittivités estimées étant inférieure à 0,01 .

teneur en eau / permittivité diélectrique / méthode non-destructive / couvert végétal / biomasse

Communicated by Thomas Schmugge (Beltsville, USA)

* Correspondence and reprints

Jerome.Helbert@bcgn.grignon.inra.fr 


\section{INTRODUCTION}

New fields in agriculture, such as precision agriculture, require the development of sensors and nondestructive methods to analyze the status of soil and plants. Knowing the water content of plants is very important since this information can be used to monitor plant growth and to estimate total biomass.

At early stages, the estimation of the nitrogen requirements of a growing crop is possible if we can infer the maximal potential biomass [2], using the dilution curve [23]. After flowering, it has been shown in the case of wheat crops that the spike layer water content is correlated to the total biomass and so to the final yield [31].

On the other hand, rapid variations of plant water content are related to hydric status and can reveal water stresses. So it is important to know the water content for monitoring irrigation.

The destructive estimation of the plant water content or the biomass by the classical gravimetric method is very long and tedious. The development of remote sensing techniques offers an efficient alternative [36].

Using optical radiometry, it has been shown that the Leaf Area Index of a crop is related to spectral vegetation indices like the NDVI $[3,5]$. The efficiency of the light interception by plants can then be estimated from the LAI [24], or directly from spectral measurements [9, $32]$. Once the efficiency of light interception has been estimated, production models such as those presented by Monteith $[25,26]$ can then be used to predict the total crop biomass $[4,22]$. The final yield can also be predicted using spectral indices $[1,7,8,17]$. Generally, the precision of the estimated yield can be evaluated to within about $10 \%$.

The plant water content is often estimated from microwave measurements. This has been done in several studies but results on crops are not accurate since there is interaction between the electromagnetic waves and the plant geometry [15]. With radar, the estimation of the water content in crops is quite difficult because of the small optical depth of the vegetation [34], while estimating forest biomass is possible and quite accurate [10, 21]. With passive radiometers, measurements are better correlated with plant water content $[18,19]$, but the best accuracy is about 15\% [37].

Previous studies aimed at the in situ biomass estimation and daily variations of water content do exist. For example, Gosse and de Parcevaux [16] have tried to estimate the plant water content using microwave horns, but the accuracy of the measurements are not good enough. Schätzler and Kuhn [30] and Batra et al. [6] have shown that the absorption of radiation can be related to the plant water content and that it can be possible to assess the vertical distribution of water in vegetation canopies. This technique is accurate but the drawback is that the use of radioactive elements is now prohibited for field measurements.

Therefore, a new instrument, an electrostatic multipole, has been developed to assess the in situ water content of vegetation canopy. It is based on an electromagnetic method, which provides non-destructive, non-contact, and instantaneous measurements. Furthermore, the electrostatic multipole is light and portable and can be easily carried within fields. The spatial range of the instrument is about $2 \mathrm{~m} \times 2 \mathrm{~m}$ so it can sample quickly the plant water content out in the field. A previous feasibility study performed on wheat crops showed that measurements obtained with a quadrupole are correlated to the spike layer water content [14].

In this paper, we perform a theoretical study of the electrostatic multipole in order to estimate the interest of using a multi-electrode configuration to make potential difference readings. We first present in detail the design and theory of the multipole and then we numerically model the response of the instrument as a function of the electromagnetic properties of crop and soil. These results are then used to develop an inversion method which can be used for estimating the spike water content as well as the stem water content.

\section{THE ELECTROSTATIC MULTIPOLE}

\subsection{Presentation of the instrument}

The instrument is an electrostatic multipole and is derived from the quadrupoles used for prospecting in geophysics [33]. Two electrodes inject an alternating current while two others allow the measurement of a voltage (Fig. 1).

The two pairs of electrodes are placed at the altitude of the studied organs in order to have the best response to their water content. The vertical distribution of the plant water content can then be assessed. Figure 2 shows the electrodes in the spike layer for the estimation of the wheat spike water content. The electrodes do not have to be in contact with the plants as the instrument is based on the propagation of an electromagnetic wave. However, as the electrodes are plunged into the vegetation volume, the multiple contacts have no consequences on the measurements as long as vegetation can be considered as a dielectric medium. The dimensions of the multipole are quite small and make the instrument easily transportable in the field: each electrode is cylindrical with a diameter of about $12 \mathrm{~cm}$ and a thickness of $5 \mathrm{~cm}$. 


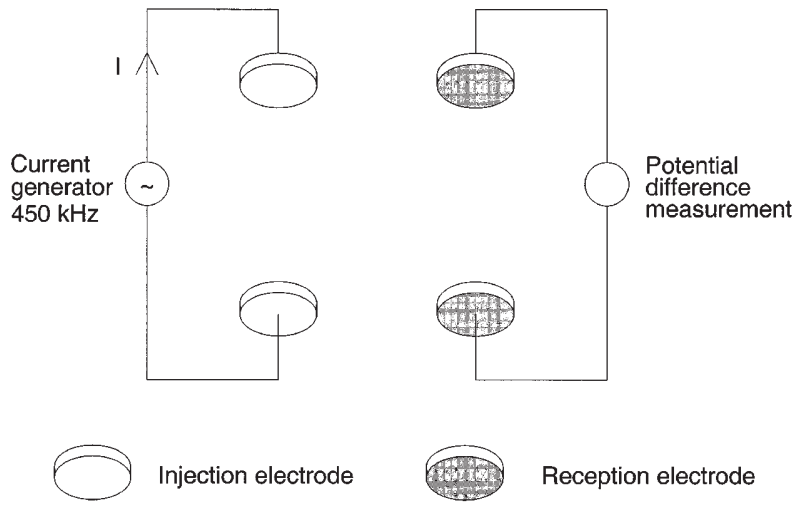

Figure 1. Electrical principle of the multipole.

The four electrodes are placed on the vertices of a $40 \times$ $40 \mathrm{~cm}^{2}$ horizontal square. Using this geometrical configuration, it is possible to make measurements at several places in a field and then to assess the horizontal distribution of the water content.

\subsection{Theoretical background}

The frequency of the injected current is $447 \mathrm{kHz}$. This frequency has been chosen between two limits: an upper frequency (about $100 \mathrm{MHz}$ ) for which there are interactions between the electromagnetic waves and the plant geometry, and a lower frequency (about $100 \mathrm{kHz}$ ) under which ionic effects are predominant, especially the Maxwell-Wagner effect [20]. At $447 \mathrm{kHz}$, the MaxwellWagner effect does exist but Féchant [12] has shown that it is very weak in comparison with the measurements made with a multipole functioning at $44 \mathrm{kHz}$. Therefore, the electromagnetic waves are supposed to be only absorbed by the constitutive water of the canopy volume. Resulting data then represents a volumetric measurement (grams of water per $\mathrm{m}^{-3}$ or $\mathrm{m}^{-2}$ ) .

The injection electrodes inject a current of intensity $I$ $(200 \mu \mathrm{A})$; the resulting electromagnetic field then interacts with the canopy, the air and the soil, and the device measures a potential difference $V$ between the reception electrodes. The ratio $V / I$ is the macroscopic impedance of the medium and is a function $F$ of the inverse of the apparent relative dielectric permittivity $\varepsilon^{*}$ :

$$
\frac{V}{I}=F\left(\frac{1}{\varepsilon^{*}}\right)
$$

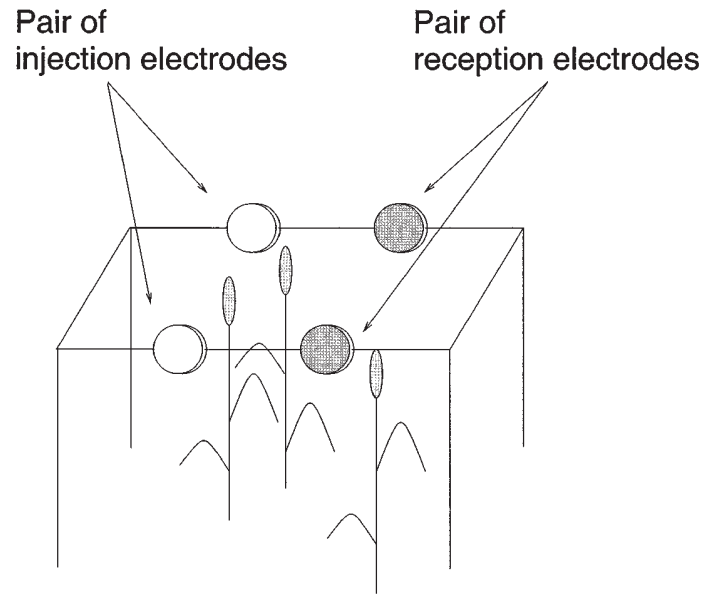

Figure 2. In situ schematic view of the multipole.

where:

$$
\begin{aligned}
\varepsilon^{*} & =\varepsilon^{\prime}-i \varepsilon^{\prime \prime} \\
& =\varepsilon^{\prime}-i \frac{\sigma}{2 \pi \varepsilon_{0} f}
\end{aligned}
$$

$\varepsilon^{\prime}$ is the real relative dielectric permittivity, $\varepsilon_{0}$ the permittivity of air $\left(\varepsilon_{0}=8.85 \cdot 10^{-12} \mathrm{~F} \cdot \mathrm{m}^{-1}\right), \sigma$ the electric conductivity $\left(\mathrm{S} \cdot \mathrm{m}^{-1}\right)$ and $f$ the frequency of the electromagnetic wave $(\mathrm{Hz})$. Thus, the signal is composed of two voltages in quadrature. The component in phase with the injected current corresponds to the imaginary part of the macroscopic permittivity while the component in quadrature with the signal corresponds to the real part of the macroscopic permittivity. As the crop canopy is a nonconducting medium (in dry atmospheric conditions), the imaginary part of the macroscopic permittivity can be ignored. Furthermore, the crop is mainly composed of air and water, and the real permittivity of water is eighty times the macroscopic permittivity of air. So, the real part of the permittivity depends mainly on the crop water content. And then, the higher the measured voltage, the lower the crop water content. Moreover, in controlled conditions, Féchant and Tabbagh [13] have measured the permittivity of a set of spikes by using a capacimeter $\left(18 \times 18 \times 2 \mathrm{~cm}^{3}\right)$ and have shown that the real dielectric permittivity at $430 \mathrm{kHz}$ is linearly correlated to the water content of the spikes.

\subsection{Experimental results}

Figure 3 represents measurements made by Féchant [12] with an initial prototype of the multipole 


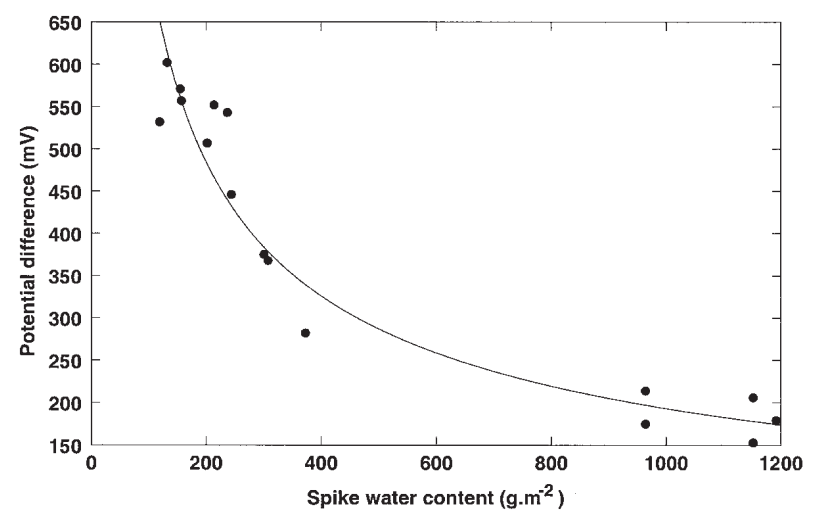

Figure 3. Correlation between measured potential differences and wheat spike water content. Adapted from Féchant [13].

instrument. These measurements are plotted against the spike water content estimated by the gravimetric technique. One can see that there is a good relationship between the potential difference and the spike water content, and that it is then possible to estimate the spike water content from the potential difference. But there is some noise that limits the accuracy of this estimation. This noise may be due to three causes: (1) the influence of the other materials like the stems and the soil, (2) the electronic noise, and (3) the uncertainty on the estimation of the spike water content by the gravimetric technique. Féchant [12] has estimated the uncertainty of the potential difference measurement due to the electronic noise to be $5 \%$.

\section{SIMULATION OF THE INSTRUMENT}

A simulation study has been performed to determine the influence of the dielectric permittivities of the various media on the potential difference measured by the instrument.

\subsection{Theoretical and computational aspects}

The frequency of the injected current is $447 \mathrm{kHz}$. It corresponds to a wavelength of $670 \mathrm{~m}$. This wavelength is very large comparatively to the dimensions of the plants. Thus, we can assume that the phase of the electromagnetic wave is constant over the scanned space. The quasi-steady state approximation can then be used and the electrostatic conditions can be applied (see appendix for details). Since we are interested in the potential outside the injection electrodes, the purpose is then to solve the Laplace equation:

$$
\Delta \mathrm{V}=0
$$

A solution of this equation is:

$$
V(\vec{r})=\frac{1}{4 \pi \varepsilon} \iiint_{v} \frac{\rho d \tau}{\left\|\vec{r}-\overrightarrow{r^{\prime}}\right\|}
$$

where $\vec{r}^{\prime}$ are the positions of the charges $\rho$ included in the volume $\mathrm{V}$.

A simplification of this equation can be achieved by considering point electrodes:

$$
V(\vec{r})=\frac{1}{4 \pi \varepsilon} \sum_{i=1}^{2} \frac{q_{i}}{\left\|\vec{r}-\vec{r}^{\prime}\right\|}
$$

where $q_{i}$ are the electric charges of the two electrodes. But the size of the electrodes is not negligible in comparison to the size of the plants. So, we have to take into account the real size of the electrodes.

Since equation (5) cannot be computed analytically we used the finite element method to numerically solve the partial differential equation [38]. The software used to compute the electrostatic potential is Modulef [11].

In the finite element method, the 3D domain of simulation has first to be meshed, and the potential is then computed on every knot of the mesh. To prevent boundary effects the domain of simulation must be much larger than the area of interest. In this case, we used a cube of $10 \mathrm{~m}$ side. The crop is modeled as four horizontal layers: the air, the spikes, the stems, and the soil. The instrument is modeled by four cylinders placed in the spike layer. Figure 4 presents a vertical section of the mesh (on this figure electrodes appear as discs). On this figure, we can see that the mesh is very fine close to the electrodes and that it becomes larger as the distance from the electrodes increases.

Since each medium is assumed to be homogeneous, a fixed real dielectric permittivity is attributed to each layer. The potential is fixed on the injection electrodes (in accordance with the injected current), and, since we assume that the electrodes are perfect conductors, we force the reception electrodes to be equipotential. We also fix the potential to zero on the limits of the simulation domain. The electrostatic potential is then calculated in the 3D space on every knot of the mesh.

Simulations are computed for one hundred couples of spike and stem permittivities; the spike permittivity 
varies within the range [1:20] while the stem permittivity varies within [1:5]. A first set of simulations (S1) has been realized with a soil permittivity fixed to 20 . A second set of simulations (S2) has been done with soil permittivities randomly chosen in the range [5:35] in order to estimate the soil contribution. Values of permittivities have been chosen in a large range to include previous measurements of permittivity made at $430 \mathrm{kHz}$ by Féchant and Tabbagh [13]. Notice that the highest values of permittivity are those of materials having the highest water contents.

\subsection{Results}

First, Table I reports the results of an analysis of variance performed on the S2 data. This analysis shows that the potential difference between the reception electrodes is mainly explained by the spike and the stem permittivities, and that the soil influence is very low, even when the electrodes are close to the ground $(40 \mathrm{~cm}$ above the soil). The Fisher's coefficients $\mathrm{F}_{0}$ are indeed very high for the spike and the stem permittivity $\left(32<\mathrm{F}_{\mathrm{o}}<390\right)$ while $\mathrm{F}_{\mathrm{o}}$ is low for the soil permittivity $\left(\mathrm{F}_{\mathrm{o}}<0.05\right)$. So, we can conclude that the soil contribution can be considered as a noise in this geometrical configuration.

Secondly, Figure 5 represents the potential difference between the reception electrodes as a function of the permittivities of the spikes and the stems (S1 data). As predicted by equation (5), the potential difference decreases as the spike and/or the stem permittivity increases. The potential difference depends both on the spike permittivity and the stem permittivity, but the stem contribution decreases when the spike permittivity increases.

Third, as a consequence of the influence of the stems, it is impossible to estimate precisely the lower values of spike water content (corresponding to the lower permittivities) from the potential differences. We have indeed two independent variables (the spike and the stem permittivities), and only one potential difference. This could

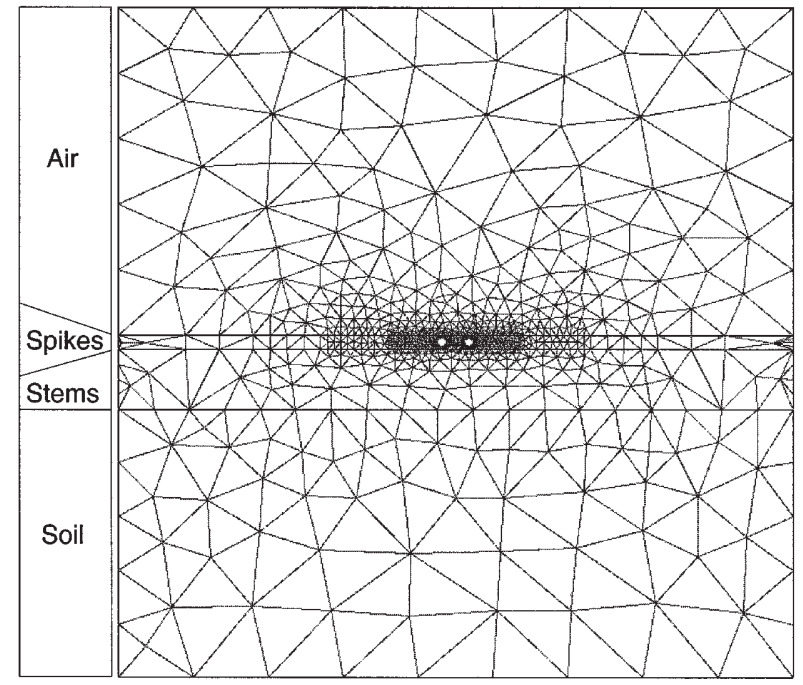

Figure 4. Vertical section of the mesh. The pairs of injection electrodes and reception electrodes are placed in the spike layer. The mesh is finest in the vicinity of the two pairs of electrodes.

explain the origin of the noise observed on the spike water content as estimated by Féchant (see Fig. 3).

The idea was thus to add a second pair of reception electrodes in order to have two simultaneous potential differences and then to be able to retrieve the spike and the stem permittivities from these two potential differences. We have tested five geometrical configurations (configurations \#1, \#2, and \#4 are reported in Fig. 6):

(1) all the electrodes in the spike layer,

(2) the new pair in the stem layer under the pair of injection electrodes,

(3) the new pair in the stem layer under the first pair of reception electrodes,

Table I. Analysis of variance showing that the potential differences $V_{1}$ and $V_{2}$ are determined by the spike and stem permittivities $\left(\varepsilon_{\text {spikes }}\right.$ and $\left.\varepsilon_{\text {stems }}\right)$ and not by the soil permittivity $\varepsilon_{\text {soil }} . V_{1}$ and $V_{2}$ are the potential differences when the multipole is $80 \mathrm{~cm}$ and $40 \mathrm{~cm}$ above the ground respectively. $\mathrm{F}_{\mathrm{o}}$ is Fisher's coefficient and $\operatorname{Pr}\left(\mathrm{F}>\mathrm{F}_{\mathrm{o}}\right)$ is the probability of having $\mathrm{F}>\mathrm{F}_{\mathrm{o}}$. The inter-electrode distance is equal to $40 \mathrm{~cm}$.

\begin{tabular}{|c|c|c|c|c|c|c|}
\hline & $\mathrm{F}_{\mathrm{o}}$ & $\operatorname{Pr}\left(\mathrm{F}>\mathrm{F}_{\mathrm{o}}\right)$ & $\mathrm{F}_{\mathrm{o}}$ & $\operatorname{Pr}\left(\mathrm{F}>\mathrm{F}_{\mathrm{o}}\right)$ & $\mathrm{F}_{\mathrm{o}}$ & $\operatorname{Pr}\left(\mathrm{F}>\mathrm{F}_{\mathrm{o}}\right)$ \\
\hline $\mathrm{V}_{1}$ & 390 & 0.0000000 & 85 & 0.0000000 & 0.03 & 0.8605228 \\
\hline $\mathrm{V}_{2}$ & 307 & 0.0000000 & 32 & 0.0000002 & 0.05 & 0.8203480 \\
\hline
\end{tabular}




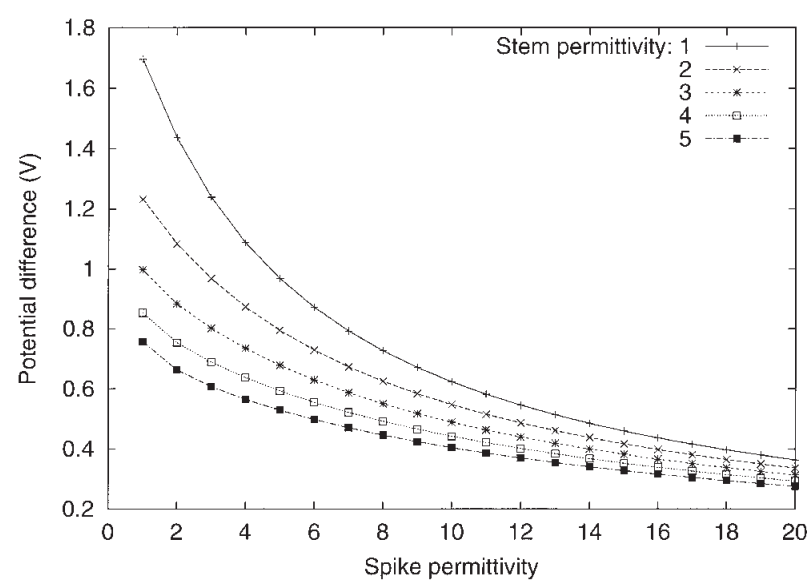

Figure 5. Potential difference between the reception electrodes as a function of the spike permittivity and the stem permittivity.

(4) the new pair in the air layer above the pair of injection electrodes,

(5) the new pair in the air layer above the first pair of reception electrodes.

In this paper, we focus on results concerning the configuration \#1 presented in Figure 6, which is a compromise between the sensitivities to the spike permittivity and the stem permittivity. As previously, we performed two sets of simulations: a first set with a soil permittivity fixed to 20 (S3 data), and a second set with random soil permittivities (S4 data). S3 data is reported in Figure 7 (the other configurations give results close to those presented in this figure).

\section{INVERSION OF THE SIMULATION DATA}

The purpose of this section is to retrieve the spike permittivity $\varepsilon$ from the potential difference data. This is theoretically possible with the instrument composed of two pairs of reception electrodes because each couple of potential differences corresponds to a unique couple of permittivities (spike and stem permittivities) (see Fig. 7).

\subsection{Results with parametric and non-linear regressions}

Among the numerous existing inversion techniques [35], we first tried to invert the simulated data (S3 and S4 data) with a classical parametric and non-linear regression method. We chose to test several models of regression curve (parabolic, hyperbolic, power laws...). Figure 8 reports the best accordance between the estimated and the exact spike permittivities. This regression was obtained with a power law model:

$$
\varepsilon_{\text {estimated }}=k V_{1}^{\alpha} V_{2}^{\beta}
$$

where $k=1310, \alpha=-5.58$, and $\beta=4.60$. Notice that these parameters, obtained by a statistical adjustment, have no particular physical significance. The correlation is good for this model $\left(r^{2}=0.9983, \sigma^{2}=0.062\right)$, but we

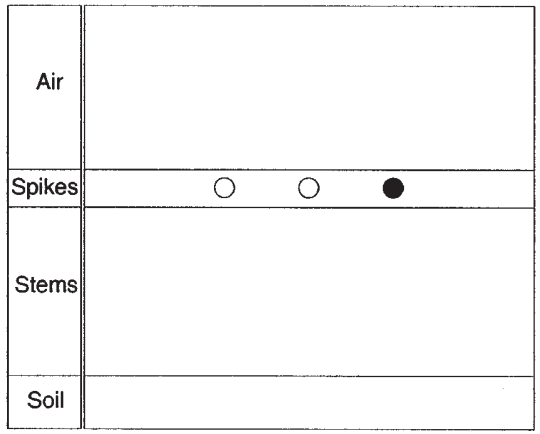

Configuration \#1

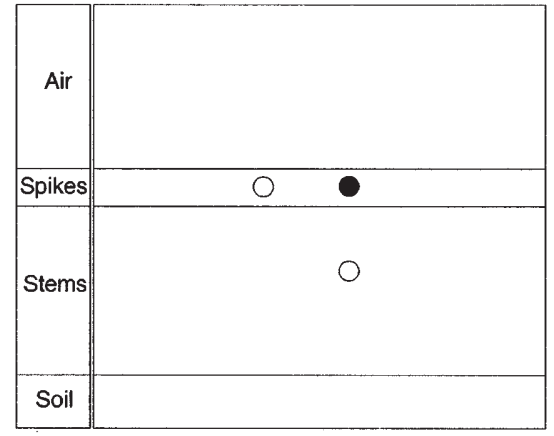

Configuration \#2

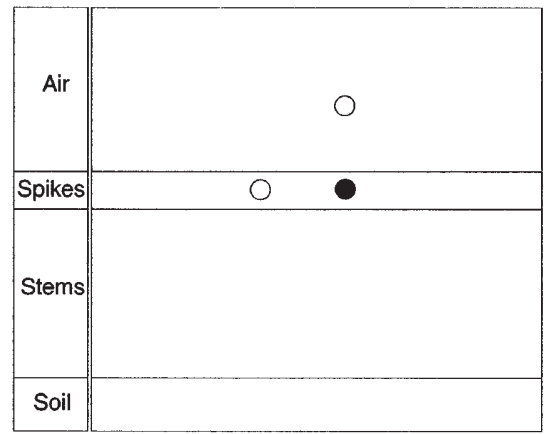

Configuration \#4

Figure 6. Presentation of three tested configurations. Configurations \#2 and \#4 improve respectively either the sensitivity to the stem permittivity or the sensitivity to the spike permittivity. Configuration \#1 is a compromise. (Injection electrode in black, reception electrodes in white.) 


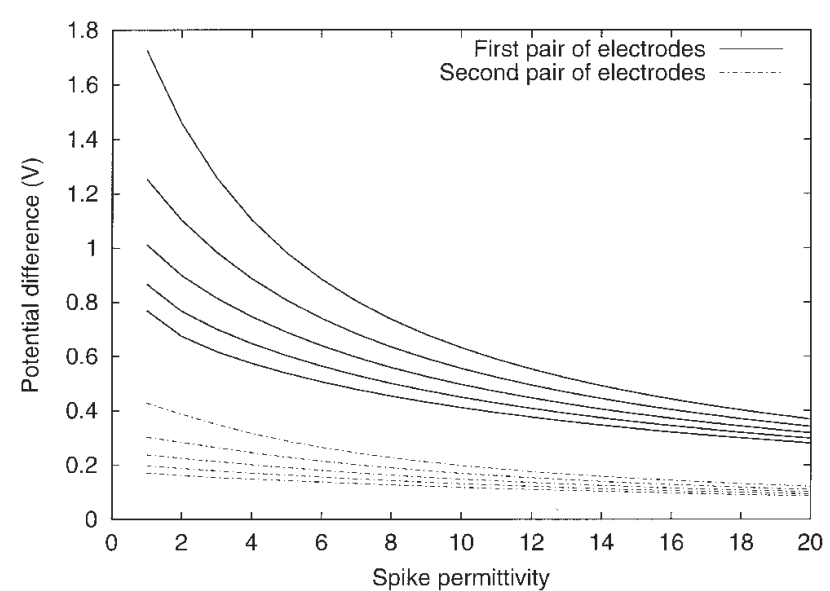

Figure 7. Potential difference between the electrodes of the first pair of reception electrodes (solid lines), and between the electrodes of the second pair of reception electrodes (dashed lines), as a function of the spike permittivity. Potential differences are plotted for five stem permittivities (stem permittivities from 1 to 5 downward).

can observe in Figure 9 the existence of a relationship between the residuals of the fitted data and the spike permittivities: they are over-estimated for the lowest and the highest values, while they are under-estimated for the mean values.

\subsection{Radial basis function neural network architecture}

In order to get better results, we have chosen to invert the data set by using an artificial neural network (ANN), which can be considered as a non-parametric regression tool [29]. More precisely, we have considered a radial basis function neural network (RBF-NN). RBF-NNs are composed of three layers: the input layer, the hidden layer, and the output layer [28, 29]. The input layer is the vector of the known potential differences and the output layer is the estimated spike permittivity (Fig. 10). Mathematically, we have:

$$
\varepsilon=\sum_{i=1}^{N} w_{i} \varphi_{i}\left(V_{1}, V_{2}\right)=\sum_{i=1}^{N} w_{i} \varphi_{i}(\boldsymbol{V}) .
$$

The purpose is to compute the values of the weight $\mathrm{w}_{\mathrm{i}}$ of each neuron, knowing the radial functions $\varphi_{i}$. The radial

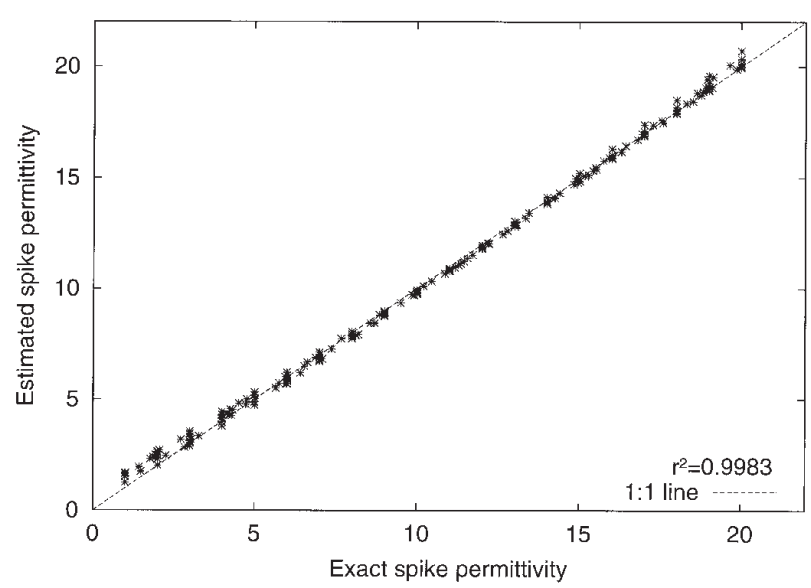

Figure 8. Comparison between the estimated spike permittivities and the exact spike permittivities. Results obtained for a non-linear regression with a power law model.

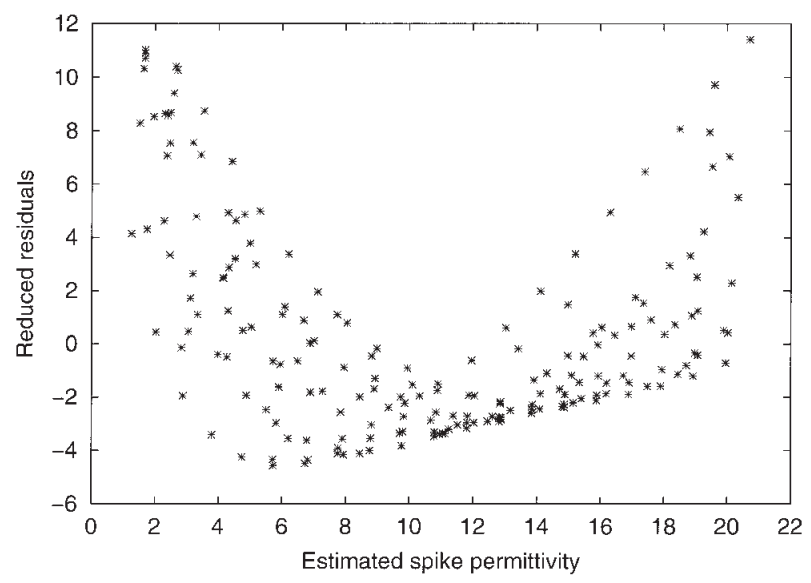

Figure 9. Plot of the reduced residuals of the estimated spike permittivities against the estimated spike permittivities showing the correlation of the residuals.

functions are chosen as Gaussian centers:

$$
\varphi_{i}(\boldsymbol{V})=\exp \left(-\frac{1}{2}\left\|\frac{\boldsymbol{V}-\boldsymbol{c}_{\boldsymbol{i}}}{\sigma_{i}}\right\|^{2}\right)
$$

where $c_{i}$ is the center of the ith basis and $\sigma_{\mathrm{i}}$ the bandwidth.

In order to compute the weights $w_{i}$ (i.e. to train the network), we implemented a forward selection algorithm 


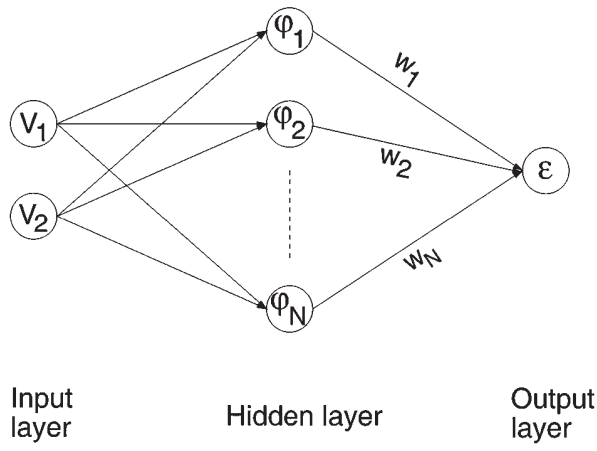

Figure 10. Architecture of the RBF neural network. The network estimates $\varepsilon$ from $V_{1}$ and $V_{2}$. Functions $\varphi_{i}$ are known, weights $w_{\mathrm{i}}$ are adjusted.

[27]. This method first selects the best centers and the best bandwidths with regard to the training data and then calculates the weights of the RBF-NN by the least squares technique. The training data used by the algorithm is the results of the 100 simulations made with a fixed soil permittivity (S3 data).

\subsection{Results with the neural network}

The accuracy of the ANN is then estimated with the test set composed of noisy data (S4 data). Results are reported in Figure 11. One can see that the accuracy of the RBF-NN is very good since the estimated values of the spike permittivity are very close to the exact permittivities introduced in the simulations $\left(r^{2}=0.99996\right.$ for the test data). The variances of the estimated spike permittivities are $\sigma^{2}=0.00029$ for the training set and $\sigma^{2}=$ 0.0054 for the test set.

The RBF-NN can also be used to estimate the stem permittivities (Fig. 12). Results are good too: $\sigma^{2}=0.00$ on the training set, and $\sigma^{2}=0.01$ on the test set $\left(r^{2}=\right.$ 0.99319 for the test data). But the accuracy of the stem permittivity estimation is less than the estimation of the spike permittivity because there are only five different values of stem permittivities in the training set, whereas there are 20 different values in the spike training set. So, the stem permittivity estimation could be better with a larger training set composed of more different values of the stem permittivities.

In conclusion, the ANN is well adapted to the estimation of the permittivity of the organs of the plants. From two potential differences, it is possible to estimate inde-

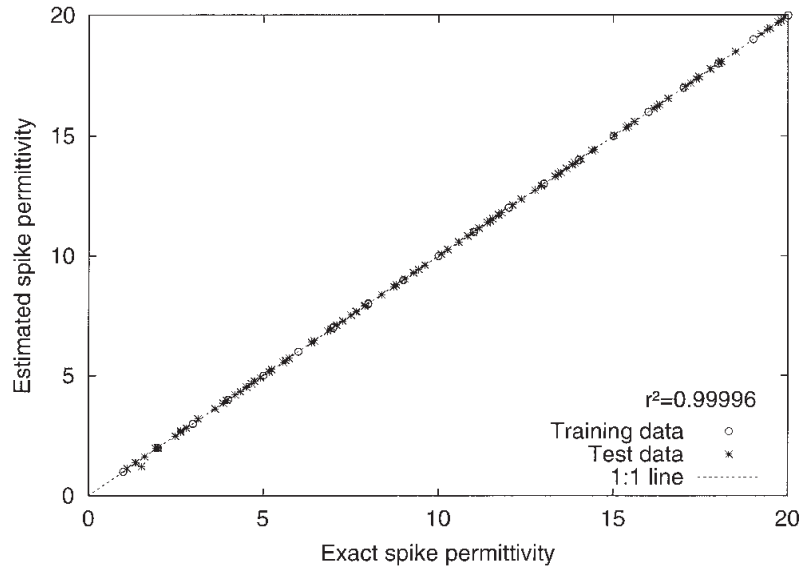

Figure 11. Comparison of the spike permittivities estimated with the RBF-NN with exact spike permittivities.

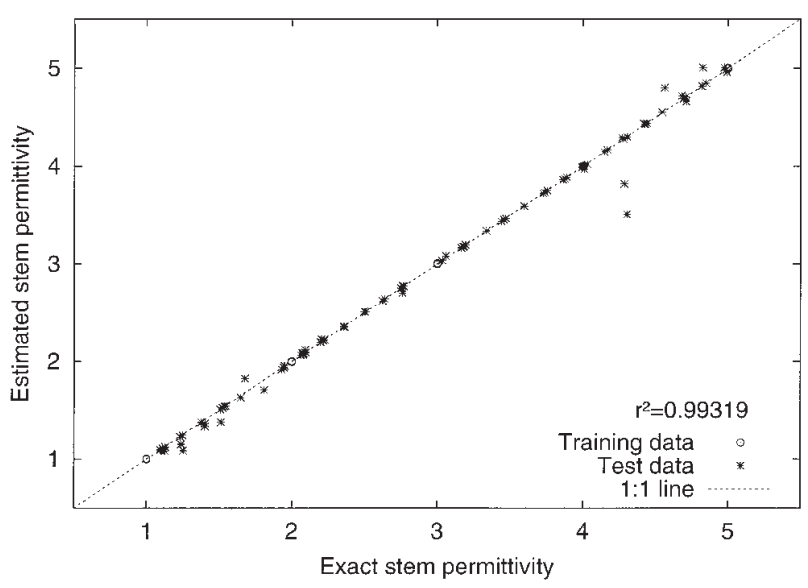

Figure 12. Comparison of the stem permittivities estimated with the RBF-NN with exact stem permittivities.

pendently and accurately the spike and the stem permittivities.

\section{DISCUSSION}

Several points have to be discussed.

\subsection{Estimation of the spike water content}

It follows the scheme:

$$
V \rightarrow \varepsilon \rightarrow w
$$


where $w$ is the spike water content $\left(\mathrm{g} \cdot \mathrm{m}^{-2}\right)$. One could remark that it would be possible - and less tedious - to estimate directly the water content from the potential difference $(V \rightarrow w)$. We would have to compare the multipole measurements with the spike water content estimated by the gravimetric technique. But this would have to be done for every configuration of the electrodes of the instrument.

From an operational point of view, we propose to measure $V$, to estimate $\varepsilon$ by using the inversion procedure, and finally to retrieve $w$.

On the first hand, roughly two parameters define the amount of water by layer: the water content of individual organs and the density of plants. This amount of water by layer, or the water profile, is then related to $\varepsilon$. Thus, we can consider that the relation $\varepsilon \rightarrow w$ is an intrinsic property of the crop, and we can assume that this relation can be inverted.

On the other hand, the second part of the model $(V \rightarrow$ $\varepsilon$ ) depends only on the geometrical configuration of the instrument.

Thus, if we want to change the configuration of the instrument to estimate more precisely the plant water content, we only need to simulate the response of the instrument in this new configuration, and then to retrieve $\varepsilon$ from the RBF-NN.

\subsection{Accuracy of the method}

We saw previously that the variance of the ANN, calculated for the test data, is $\sigma^{2}=0.0054$.

This is quite a good result but it is limited by the accuracy of the measurement of the potential difference. The uncertainty $\Delta V$ of the instrument, estimated from the measurements made by Féchant [12], is about 5\%. If we add a noise of level $5 \%$ on the test data (S4), the accuracy of the ANN reaches 10\%; this can be seen in Figure 13 where most of the estimated spike permittivities lie within the $\pm 10 \%$ error interval. Thus, the absolute error on the spike permittivity estimation varies between 0.1 and 2 .

We then have to consider the second part of the model: $\varepsilon \rightarrow w$. We can assume a linear relation between the spike water content and the permittivity:

$$
w=a \varepsilon+b .
$$

To build this relation, the water content is estimated by the gravimetric technique, and the coefficients $a$ and $b$ are computed by regression. Once $a$ and $b$ are calculated, the linear relation can be used to estimate the water con-

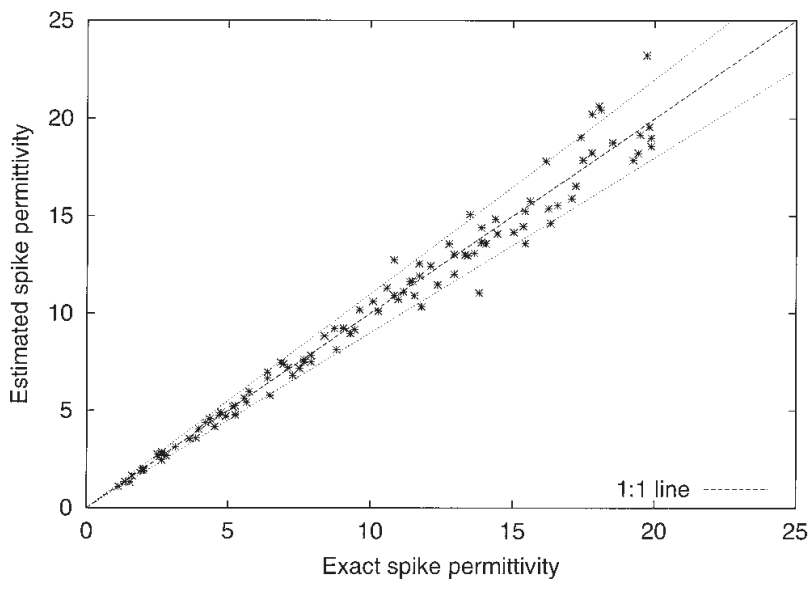

Figure 13. Spike permittivities estimated with the RBF-NN from noisy potential differences. Comparison with exact spike permittivities and $\pm 10 \%$ error intervals.

tent from the permittivity. The uncertainty $\Delta w$ on the water content is then:

$$
\begin{aligned}
\Delta w & =\frac{\partial w}{\partial \varepsilon} \Delta \varepsilon+\frac{\partial w}{\partial a} \Delta a+\Delta b \\
& =a \Delta \varepsilon+\underbrace{\frac{\partial w}{\partial a} \Delta a+\Delta b}_{\Delta w_{r g}} .
\end{aligned}
$$

$\Delta w_{r e g}$ is the error on the estimation of the water content by the gravimetric technique. $\Delta w_{\text {reg }}$ is generally about $10 \%$, corresponding to $\pm 10 \mathrm{~g} \cdot \mathrm{m}^{-2}$ for the lower spike water contents, and $\pm 150 \mathrm{~g} \cdot \mathrm{m}^{-2}$ for the highest spike water contents.

We then have to compare $a \Delta \varepsilon$ to $\Delta w_{r e g}$. If we assume that a difference of permittivity of 20 represents a variation of water content of $1000 \mathrm{~g} \cdot \mathrm{m}^{-2}$ (compare Figs. 3 and 7), we have $a=\frac{1000}{20}=50$, and finally:

$$
\begin{gathered}
10<\Delta \mathrm{w}_{\text {reg }}<150 \mathrm{~g} \cdot \mathrm{m}^{-2} \\
5<\mathrm{a} \Delta \varepsilon<100 \mathrm{~g} \cdot \mathrm{m}^{-2} .
\end{gathered}
$$

The main source of error is then included in the relation between the water content and the permittivity because of the relative uncertainty on the gravimetric technique. The proposed multipole method can therefore be considered to be more precise than the gravimetric method which is the classical destructive method. 


\subsection{Effect of the soil conductivity}

We have shown that the soil permittivity has no influence on the potential differences. But we have not taken the soil conductivity $\sigma$ into account (see Eq. (3)). Including a conductivity in the soil permittivity leads us to create a phase component $\left(V_{\mathrm{P}}\right)$ and can alter the quadrature component $\left(V_{\mathrm{Q}}\right)$ of the potential difference. We have then simulated the response of the instrument for a two layer medium composed of air and soil. Soil permittivities varied from 5 to 30 and soil conductivities were chosen between $10^{-4}$ and $10^{-1} \mathrm{~S} \cdot \mathrm{m}^{-1}$, including most of the observed values. Simulations were performed for several electrode altitudes: 0.4, 0.6, 0.8, and $1 \mathrm{~m}$ above the ground (S5 data). Simulations without introducing soil conductivity (with only real soil permittivities) were also performed (S6 data).

Concerning the phase component, the values of $V_{\mathrm{P}}$ lie between 0 and $30 \mathrm{mV}$, which are low values in comparison with the quadrature component (about $1 \mathrm{~V}$ ).

Concerning the quadrature component, the values $V_{\mathrm{Q}}$ of S5 data are close to the values $V_{\mathrm{Q}}$ of S6 data and relative variations are comparable: we can see in Table II that taking the soil conductivity into account increases the range of variation of the quadrature component but the effect is very weak even when the electrodes are $40 \mathrm{~cm}$ above the ground. For the other altitudes the effect is negligible.

Thus, in these electrode geometrical configurations, it is possible to neglect the soil conductivity as we are not interested in the phase component of the potential differences.

\subsection{Variation of the injected current}

The injected current could be changed to adjust the signal to the volume we want to scan. Theoretically, multiplying the injection current by a factor increases the range of the instrument by the same factor. But in practice, there are two limitations to the modification of the current: too much intensity would reduce the life of the batteries, while a current chosen too low would reduce the signal to noise ratio.

\subsection{Presence of liquid water on vegetation}

As a last remark, we can note that any variation of the water present in the volume may influence the electrostatic measurement. Especially, variations of measurement conditions must be taken into account, such as the presence of dew or rainwater on the plant organs and the ground.

\section{CONCLUSION}

As the plant water content can be directly related to the standing fresh biomass, its measurement is one of the ways for monitoring and mapping the crop status and development. The reference technique is the classical gravimetric method which is destructive, time consuming and can only be applied to a limited number of samples.

The electrostatic multipole developed offers a new way to assess the crop water content in field conditions. The theoretical analysis as well as the preliminary experimental results show the potential of this instrument. The simulation performed on a wheat crop shows the possibility of a simultaneous assessment of the water content of spike and stem layers with an accuracy comparable or even better than that given by the gravimetric technique.

The multipole coupled with a geographic positioning system (GPS) could then be used for mapping the crop water content at the field scale for monitoring the crop

Table II. Absolute and relative variations of the quadrature component of the potential differences $\left(V_{\mathrm{Q}}\right)$ calculated on S5 data (simulation including soil conductivities), and S6 data (simulations with real soil permittivities only). Values computed for several electrode altitudes.

\begin{tabular}{|c|c|c|c|c|c|}
\hline & & $40 \mathrm{~cm}$ & $60 \mathrm{~cm}$ & $80 \mathrm{~cm}$ & $1 \mathrm{~m}$ \\
\hline Absolute variation & with soil conductivity (S5 data) & 50.3 & 17.9 & 7.17 & 3.37 \\
\hline of $V_{\mathrm{Q}}(\mathrm{mV})$ & without soil conductivity (S6 data) & 20.3 & 13.0 & 5.98 & 3.05 \\
\hline Relative variation & with soil conductivity (S5 data) & 3.9 & 1.3 & 0.49 & 0.23 \\
\hline of $V_{\mathrm{Q}}(\%)$ & without soil conductivity (S6 data) & 2.0 & 1.0 & 0.46 & 0.22 \\
\hline
\end{tabular}


growth and its heterogeneity and assessing the final yield. This technique then offers a good potential in precision farming and also in providing ground truth information for calibrating remote sensing data. However, it is now necessary, after this feasibility study, to test the multipole on several crops in order to evaluate the effective accuracy of this new measurement technique in field conditions.

\section{Appendix: Some fundamentals of electromagnetism}

The two first equations of Maxwell with no temporal dependance are:

$$
\begin{gathered}
\vec{\nabla} \cdot \vec{D}=\rho \\
\vec{\nabla} \times \vec{E}=0
\end{gathered}
$$

where $\vec{D}$ is the electric induction, $\vec{E}$ the electric field $(\vec{D}=\varepsilon \vec{E})$, and $\rho$ the electric charges. From equation(14), a function $V$ exists, such as $\vec{E}=-\vec{\nabla} V$, because the equality $\vec{\nabla} \times(\vec{\nabla} V)=0$ is always true whatever $V$. In electromagnetics, $V$ is the electrostatic potential. Equation (13) then becomes:

$$
\begin{aligned}
\vec{\nabla} \cdot \vec{D} & =\vec{\nabla} \cdot(\varepsilon \vec{E}) \\
& =-\vec{\nabla} \cdot(\varepsilon \vec{\nabla} V) \\
& =-\vec{\nabla} \varepsilon \cdot \vec{\nabla} V-\varepsilon \vec{\nabla} \cdot(\vec{\nabla} V) \\
& =-\vec{\nabla} \varepsilon \cdot \vec{\nabla} V-\varepsilon \Delta V=\rho .
\end{aligned}
$$

But $\vec{\nabla} \varepsilon=0$ since we assume an homogeneous medium. We then obtain Poisson's equation:

$$
\Delta V=-\frac{\rho}{\varepsilon} .
$$

Furthermore, $\rho \neq 0$ on the electrodes and $\rho=0$ everywhere else.

Acknowledgments: The authors would like to thank Dr. M. Vidrascu (INRIA, Institut National de la Recherche en Informatique et Automatique, Rocquencourt, France), Prof. O. Picon (University of Marne-la-Vallée, France), Prof. A. Tabbagh (University of Paris VI, France), Prof. Ph. Huet (INA P-G, Institut National Agronomique Paris-Grignon, France), and Dr. J.-P. Wigneron (INRA, Institut National de la Recherche Agronomique, Montfavet, France) for their help with this work.

Financial support for this work was partly provided by the INRA Precision Agriculture Project 1999-2000.

\section{REFERENCES}

[1] Aase J.K., Siddoway F.H., Assessing winter wheat dry matter production via spectral reflectance measurements, Remote Sens. Environ. 11 (1981) 267-277.

[2] Akkal N., Jeuffroy M.H., Meynard J.M., Boissard P., Helbert J., Valéry P., Lewis P., Assessment of a method for estimating the nitrogen requirements of a wheat crop based on an early estimate of cover fraction, in: Stafford J.V. (Ed.), Precision Agriculture '97. Vol. I: Spatial variability in soil and crop, 1st European Conference, Fertiliser Society, Warwick, UK, 07-10/09/1997, Bios Scientific Publ., Oxford, pp. $405-412$.

[3] Asrar G., Fuchs M., Kanemasu E.T., Hatfield J.L., Estimating absorbed photosynthetic radiation and leaf area index from spectral reflectance in wheat, Agron. J. 76 (1984) 300-306.

[4] Asrar G., Kanemasu E.T., Jackson R.D., Pinter P.J., Estimation of total above-ground phytomass production using remotely sensed data, Remote Sens. Environ. 17 (1985) 211-220.

[5] Asrar G., Kanemasu E.T., Yoshida M., Estimates of leaf area index from spectral reflectance of wheat under different cultural practices and solar angle, Remote Sens. Environ. 17 (1985) 1-11.

[6] Batra R.K., Singh B., Singh K., Determination of water content of plant leaves by beta attenuation, Int. J. Rad. Appl. Instr., Part A: Appl. Rad. Isotopes 43 (1992) 1235-1239.

[7] Benedetti R., Rossini P., On the use of NDVI profiles as a tool for agricultural statistics: The case study of wheat yield estimate and forecast in Emilia Romagna, Remote Sens. Environ. 45 (1993) 311-326.

[8] Das D.K., Mishra K.K., Kalra N., Assessing growth and yield of wheat using remotely-sensed canopy temperature and spectral indices, Int. J. Remote Sens. 14 (1993) 3081-3092.

[9] Daughtry C.S.T., Gallo K.P., Bauer M.E., Spectral estimates of solar radiation intercepted by corn canopies, Agron. J. 75 (1983) 527-531.

[10] Dobson M.C., Ulaby F.T., Le Toan T., Beaudoin A., Kasischke E.R., Christensen N., Dependance of radar backscatter on coniferous forest biomass, IEEE Trans. Geosci. Remote Sens. 30 (1992) 412-415.

[11] du Toit H.F., George P.L., Lang P., Paté P., Steer D., Vidrascu M., An introduction to Modulef. Modulef user guide \#1, INRIA, 1991, 309 p. (available at http://wwwrocq.inria.fr/modulef/Doc/GB/welcome.html.)

[12] Féchant C., Réalisation d'un quadripôle de mesure in situ de la permittivité diélectrique des végétaux. Première application à la détermination du contenu en eau des épis de blé, Ph.D. thesis, Université de Paris VI, 1996, 190 p.

[13] Féchant C., Tabbagh A., Mesure en laboratoire de la permittivité diélectrique moyenne fréquence de végétaux à $430 \mathrm{kHz}$ à l'aide d'un capacimètre. Relation entre permittivité apparente d'un ensemble d'épis de blé et leur contenu en eau (Laboratory measurement of the dielectric permittivity of vegetals at $430 \mathrm{kHz}$. Correlation between apparent permittivity of a 
set of wheat ears and their water content), C. R. Acad. Sci., Sér. II, fasc. B: Méc., Phys., Astron. 327 (1999) 285-298.

[14] Féchant C., Buis J.-P., Tabbagh A., In situ measurement of medium-frequency apparent permittivity using an electrostatic quadrupole. Application to the determination of the water content of wheat, Meas. Sci. Technol. 10 (1999) 174-181.

[15] Ferrazzoli P., Paloscia S., Pampaloni P., Schiavon G., Solimini D., Coppo P., Sensitivity of microwave measurements to vegetation biomass and soil moisture content, IEEE Trans. Geosci. Remote Sens. 30 (1992) 750-756.

[16] Gosse G., de Parcevaux S., Application de l'absorption des ondes centimétriques à la mesure de l'humidité des végétaux, in: Techniques d'étude des facteurs physiques de la biosphère, INRA (Eds.), 1970, pp. 359-369.

[17] Hatfield J.L., Remote sensing estimators of potential and actual crop yield, Remote Sens. Environ. 13 (1983) 301-311.

[18] Jackson T.J., O'Neill P.E., Attenuation of soil microwave emission by corn and soybeans at 1.4 and $5 \mathrm{GHz}$, IEEE Trans. Geosci. Remote Sens. 28 (1990) 978-980.

[19] Jackson T.J., Schmugge T.J., Vegetation effects on the microwave emission of soils, Remote Sens. Environ. 36 (1991) 203-212.

[20] Kittel Ch., Introduction to solid state physics, John Wiley \& Sons, 7th ed., 1996, 673 p.

[21] Le Toan T., Beaudoin A., Riom J., Guyon D., Relating forest biomass to SAR data, IEEE Trans. Geosci. Remote Sens. 30 (1992) 403-411.

[22] Leblon B., Guerif M., Baret F., The use of remotely sensed data in estimation of PAR use efficiency and biomass production of flooded rice, Remote Sens. Environ. 38 (1991) 147-158.

[23] Lemaire G., Salette J., Relation entre dynamique de croissance et dynamique de prélèvement d'azote pour un peuplement de graminées fourragères. I. Étude de l'effet du milieu (Relationship between growth and nitrogen uptake in a pure grass stand. I. Environmental effects), Agronomie 4 (1984) 423-430.

[24] Linvill D.E., Dale R.F., Hodges H.F., Solar radiation weighting for weather and corn growth models, Agron. J. 70 (1978) 257-263.

[25] Monteith J.L., Solar radiation and productivity in tropical ecosystems, J. Appl. Ecol. 9 (1972) 747-766.
[26] Monteith J.L., Climate and the efficiency of crop production in Britain, Philos. Trans. Roy. Soc. London B 281 (1977) 277-294.

[27] Orr M.J.L., Introduction to radial basis function networks, Tech. report, Centre for Cognitive Science, University of Edinburgh, Scotland, 1996, 67 p. (available at http://www.anc.ed.ac.uk/ mjo/papers/intro.ps.gz.)

[28] Poggio T., Girosi F., Networks for approximation and learning, Proc. IEEE 78 (1990) 1481-1497.

[29] Sarle W.S., Neural networks and statistical models, in: Proceedings of the nineteenth annual SAS User Group international conference, 1994, pp. 1538-1550. (available at ftp://ftp.sas.com/pub/sugi19/neural/neural1.ps.)

[30] Schätzler H.P., Kühn W., Growth studies on plant plots by gamma scanning, Int. J. Appl. Rad. Isotopes 28 (1977) 645-652.

[31] Schnyder H., Baum U., Growth of the grain of wheat (Triticum aestivum L.). The relationship between water content and dry matter accumulation, Eur. J. Agron. 1 (1992) 51-57.

[32] Steinmetz S., Guérif M., Delécolle R., Baret F., Spectral estimates of the absorbed photosynthetically active radiation and light-use efficiency of a winter wheat crop subjected to nitrogen and water deficiencies, Int. J. Remote Sens. 11 (1990) 1797-1808.

[33] Tabbagh A., Hesse A., Grard R., Determination of electrical properties of the ground at shallow depth with an electrostatic quadrupole: Field trials on archaeological sites, Geophys. Prospect. 41 (1993) 579-597.

[34] Taconet O., Benallegue M., Vidal-Madjar D., Prévot L., Dechambre M., Normand M., Estimation of soil and crop parameters for wheat from airborne radar backscattering data in C and X bands, Remote Sens. Environ. 50 (1994) 287-294.

[35] Tarantola A., Inverse problem theory. Methods for data fitting and model parameter estimation, Elsevier, 1987, 613 p.

[36] Tucker C.J., A critical review of remote sensing and other methods for non-destructive estimation of standing biomass, Grass Forage Sci. 35 (1980) 177-182.

[37] Wigneron J.-P., Chanzy A., Calvet J.-C., Bruguier N., A simple algorithm to retrieve soil moisture and vegetation biomass using passive microwave measurements over crop fields, Remote Sens. Environ. 51 (1995) 331-341.

[38] Zienkiewicz O., The finite element method, McGrawHill, Maidenhead, England, 1977, 787 p.

To access this journal online: www.edpsciences.org 